\title{
Bounds for the Two-Dimensional Discrete Harmonic Green's Function
}

\author{
By Moshe Mangad
}

\begin{abstract}
Estimation of bounds for the two-dimensional discrete harmonic Green's function is obtained. These estimations can then be used to obtain approximate solutions to two-dimensional boundary problems associated with the harmonic difference operator.
\end{abstract}

1. Introduction. In dealing with partial differential operators, it is desirable to obtain the "free-space" Green's function (valid in the entire space under consideration) for the Laplacian operator, $\Delta$, which permits writing solutions for boundary value problems in integral form [4].

A similar situation exists in solving boundary value problems associated with partial difference operators. It is desirable to find the analog free-space discrete harmonic Green's function $g(m, n)$, which permits writing solutions in summation form for the boundary value problems of the difference equations [1]-[3].

Unlike the two-dimensional continuous case, where the Green's function is known to be $\log r$, an exact estimate for $g(m, n)$ (where $m$ and $n$ are integers and where the mesh widths are unity) is not available (see [3] and [6]). This is because the evaluation involves an elliptic integral (see Eqs. (6) and (7)); only asymptotic estimates for $g(m, n)$ are known.

In this paper, we obtain explicit bounds (see Theorem 1 ) for $g(m, n$ ) which yield very reasonable numerical estimates for intermediate values of $m$ and $n$. Then, by making a suitable transformation, similar results are obtained for the discrete harmonic Green's function $g_{p}(Q)$, associated with mesh widths $h$ in $x$ and $y(x=m h$, $y=n h)$.

2. Known Results. Let $D$ be the harmonic difference operator; i.e.,

$$
\begin{gathered}
D u(m, n)=u(m+1, n)+u(m-1, n)+u(m, n+1) \\
+u(m, n-1)-4 u(m, n),
\end{gathered}
$$

where $m$ and $n$ are integers. Then, $g(m, n)$ is defined [2] as the unique solution of

$$
\begin{aligned}
D g(m, n) & =0, \quad \text { except at }(0,0) \\
D g(0,0) & =-1 \\
g(0,0) & =0
\end{aligned}
$$

the first differences of $g(m, n) \rightarrow 0$ as $k=\left(m^{2}+n^{2}\right)^{1 / 2} \rightarrow \infty$.

Duffin and Shaffer [3] showed, by means of an operational calculus based on Fourier series, that

$$
g(m, n)=\frac{1}{(2 \pi)^{2}} \int_{-\pi}^{\pi} \int_{-\pi}^{\pi} \frac{1-\exp [i(m x+n y)]}{4\left(\sin ^{2} x / 2+\sin ^{2} y / 2\right)}
$$

Received November 30, 1964. 
On the other hand, McCrea and Whipple [6] showed, by considering a two-dimensional random walk problem, that

$$
g(m, n)=\frac{1}{2 \pi} \int_{0}^{\pi} \frac{1-\exp [-|m| y] \cos n x}{\sinh y} d x .
$$

with

$$
\cos x+\cosh y=2 .
$$

The asymptotic estimates obtained by [3] and [6] are, respectively

$$
g(m, n)=\frac{1}{2 \pi}\left[\log k+\frac{3}{2} \log 2+\gamma\right]+O\left(\frac{1}{k^{2}}\right)
$$

$$
\text { as } k=\left(m^{2}+n^{2}\right)^{1 / 2} \rightarrow \infty \text {. }
$$

$$
g(m, n)=\frac{1}{2 \pi}\left[\log k+\frac{3}{2} \log 2+\gamma\right]+o\left(\frac{1}{k}\right) \quad \text { as } k \rightarrow \infty .
$$

Here $\gamma$ is the Euler's constant.

3. Statement of Main Results. The main results of this paper are contained in the following theorem, which will be proved in Section 5 .

THEOREM 1. If $k^{2}=m^{2}+n^{2} \neq 0$ and the mesh widths are unity (i.e., $m, n=0$, $\pm 1, \pm 2$, etc. $)$, then

$$
\frac{-53 \cdot 6}{k^{2}} \leqq 2 \pi g(m, n)-\log k-\frac{3}{2} \log 2-\gamma \leqq \frac{1}{12 k^{2}}+\frac{53 \cdot 6}{k^{2}} .
$$

Let $P=\left(x_{P}, y_{P}\right), Q=\left(x_{Q}, y_{Q}\right)$, and $m=\left(x_{Q}-x_{P}\right) / h, n=\left(y_{Q}-y_{P}\right) / h$. If $\rho=\overline{P Q}=\sqrt{ }\left(\left(x_{Q}-x_{P}\right)^{2}+\left(y_{Q}-y_{P}\right)^{2}\right)$, then the bounds for the Green's functions $g_{P}(Q)$ associated with mesh widths $h$ are

$$
\frac{53 \cdot 6 h^{2}}{\rho^{2}} \leqq 2 \pi g_{P}(Q)-\log \rho-\frac{3}{2} \log 2-\gamma \leqq \frac{53 \cdot 6 h^{2}}{\rho^{2}}+\frac{h^{2}}{12 \rho^{2}}, \rho \geqq h>0 .
$$

Remark. As will be shown later, estimates (11) could be improved to also contain terms of the form $O\left(1 / k^{4}\right)$ or $O\left(1 / \rho^{4}\right), O\left(1 / k^{6}\right)$ or $O\left(1 / \rho^{6}\right)$, etc.

4. Preliminary Results and Lemmas. To obtain explicit bounds for $g(m, n)$, we shall use representation (7) with the two properties:

$$
g(m, n)=g(n, m)
$$

and

$$
g(m, m)=\frac{1}{\pi} \sum_{j=1}^{m} \frac{1}{2 j-1}
$$

obtained in [6]. Next, one notes from Eq. (7) that $g(m, n)=g(m,-n)$ and, hence

$$
g(m, n)=g(m,-n)=g(n, m)=g(n,-m)=g(-m, n)=g(-m,-n) .
$$

This means that it is sufficient to consider the behavior of $g(m, n)$ for $m \geqq n \geqq 0$ and, without loss of generality, assume that $m \geqq 1$. In addition, for any given $x$, 
we shall consider only the non-negative values of $y$ determined by relation (8).

From Eq. (7),

$$
2 \pi[g(m, n)-g(m, m)]=\int_{0}^{\pi} \frac{(\cos m x-\cos n x)}{\sinh y} e^{-m y} d x .
$$

When $m$ is large, the important part of the integral above occurs with small values of $x$. Accordingly, we divide the range of integration at $\epsilon$, defined by $\epsilon=m^{-1 / 3}$. Next, define

$$
\begin{aligned}
& A=\int_{0}^{\infty} \frac{(\cos m x-\cos n x)}{x} e^{-m x} d x \\
& B=\int_{\epsilon}^{\infty} \frac{(\cos m x-\cos n x)}{x} e^{-m x} d x ; \\
& C=\int_{\epsilon}^{x} \frac{(\cos m x-\cos n x)}{\sinh y} e^{-m y} d x \\
& D=\int_{0}^{\epsilon}[\cos m x-\cos n x]\left[\frac{e^{-m y}}{\sinh y}-\frac{e^{-m x}}{x}\right] d x .
\end{aligned}
$$

Then, by relation (15) and by letting $A^{*}=A+2 \pi g(m, n)$, there results

$$
A^{*}-|B|-|C|-|D| \leqq 2 \pi g(m, n) \leqq A^{*}+|B|+|C|+|D| \text {. }
$$

4.1. Bounds for $A^{*}$ and $|B|$. By using Laplace transforms,

$$
A=\frac{1}{2} \log \left(m^{2}+n^{2}\right) / 2 n^{2} .
$$

Next, it is well known [5] that

$$
\sum_{j=1}^{n} \frac{1}{2 j-1}=\frac{1}{2}(\gamma+\log n)+\log 2+\frac{B_{1}}{8 n^{2}}-\frac{\left(2^{3}-1\right) B_{2}}{64 n^{4}}+\cdots,
$$

where the $B_{j}$ 's are Bernouli-type numbers given by $B_{1}=\frac{1}{6}, B_{2}=\frac{1}{30}$, etc. Combining results, therefore, we have the following bounds for $A^{*}$ :

$$
0 \leqq A^{*}-\log k-\frac{3}{2} \log 2-\gamma \leqq \frac{1}{24 m^{2}}, \quad m \geqq 1 .
$$

Finally, by recalling $B$ from Eq. (16), it is easily deduced that

$$
|B| \leqq 2 m^{-2 / 3} \exp \left[-m^{2 / 3}\right], \quad m \geqq 1 .
$$

4.2. Bounds for $|C|$. To find bounds for $|C|$, the following lemma is needed:

Lemma 1. If $\epsilon \leqq x \leqq \pi$, where $0<\epsilon \leqq 1$, then $y>9 \epsilon / 10$.

Proof. By considering the series for $\cosh y=2-\cos x$ and $\cosh 9 \epsilon / 10$, it can easily be shown that $\cosh y>\cosh 9 \epsilon / 10$, so that $y>9 \epsilon / 10$.

Using Lemma 1 and the fact that $1 / \sinh y<1 / y<10 / 9 \epsilon$ for $0<\epsilon \leqq 1, \epsilon \leqq$ $x \leqq \pi$, one obtains, after neglecting negative valued terms,

$$
|C|<\frac{20}{9} \pi m^{1 / 3} \exp \left[-9 m^{2 / 3} / 10\right], \quad m \geqq 1 .
$$


4.3. Bounds for $|D|$. To obtain bounds for $|D|$, the intermediary inequalities contained in the following lemma are needed:

LEMma 2. If $0 \leqq x \leqq 1$, then

$$
\begin{aligned}
x & \geqq y \geqq x-x^{3} / 10 \\
\sinh y & \geqq 9 x / 10 \\
\sinh x & \leqq 6 x / 5 \\
\cosh x & <8 / 5 .
\end{aligned}
$$

Proof. $\cosh y=1+y^{2} / 2 !+y^{4} / 4 !+\cdots \geqq 1+y^{2} / 2$ and $\cosh y=2-$ $\cos x \leqq 1+x^{2} / 2$. Therefore, $y \leqq x$. Next,

$$
\cosh y=2-\cos x \geqq 1+\frac{x^{2}}{2}-\frac{x^{4}}{24}
$$

and

$$
\begin{gathered}
\cosh \left(x-\frac{x^{3}}{10}\right)-1 \equiv \cosh t-1=\frac{t^{2}}{2}+\frac{t^{4}}{24}\left[1+\frac{t^{2}}{5 \cdot 6}+\frac{t^{4}}{5 \cdot 6 \cdot 7 \cdot 8}+\cdots\right] \\
\leqq \frac{t^{2}}{2}+\frac{t^{4}}{24}\left[1+\frac{t^{2}}{5^{2}}+\frac{t^{4}}{5^{4}}+\cdots\right]=\frac{t^{2}}{2}+\frac{25 t^{4}}{24\left(25-t^{2}\right)}=\cdots \\
=\frac{x^{2}}{2}+\frac{x^{6}-20 x^{4}}{200}+\frac{10^{4} x^{4}-4000 x^{6}+600 x^{8}-40 x^{10}+x^{12}}{96\left[2500-100 x^{2}+20 x^{4}-x^{6}\right.} \leqq \cdots \leqq \frac{x^{2}}{2}-\frac{9 x^{4}}{200} .
\end{gathered}
$$

Hence

$$
\cosh \left(x-\frac{x^{3}}{10}\right) \leqq 1+\frac{x^{2}}{2}-\frac{9 x^{4}}{200} \leqq 1+\frac{x^{2}}{2}-\frac{x^{4}}{24} \leqq \cosh y
$$

and, therefore

$$
y \geqq x-\frac{x^{3}}{10}
$$

Now

$$
\sinh y \geqq y \geqq x-\frac{x^{3}}{10} \geqq x-\frac{x}{10}=\frac{9 x}{10}
$$

Therefore,

$$
\begin{aligned}
\sinh x & =x+\frac{x^{3}}{3 !}+\frac{x^{5}}{5 !}+\cdots \leqq x+\frac{x}{3 !}+\frac{x}{5 !}+\cdots \\
& =x \sinh 1=\frac{x\left(e^{2}-1\right)}{2 \epsilon} \leqq \frac{6 x}{5}
\end{aligned}
$$

Finally

$$
\cosh ^{2} x=1+\sinh ^{2} x \leqq 1+\frac{36 x^{2}}{25} \leqq 1+\frac{36}{25}=\frac{61}{25}
$$


so that

$$
\cosh x<\frac{8}{5}
$$

This completes the proof of Lemma 2.

Now, from Eq. (16)

$$
|D| \leqq 2 \int_{0}^{\epsilon}\left|\frac{e^{-m y}}{\sinh y}-\frac{e^{-m x}}{x}\right| d x
$$

However

$$
\begin{aligned}
\left|\frac{e^{-m y}}{\sinh y}-\frac{e^{-m x}}{x}\right| & =\left|\frac{e^{-m y}}{\sinh y}-\frac{e^{-m x}}{\sinh x} \frac{\sinh x}{x}\right| \\
& =\left|\frac{e^{-m y}}{\sinh y}-\frac{e^{-m x}}{\sinh x}\left(1+\frac{x^{2}}{3 !}+\frac{x^{4}}{5 !}+\cdots\right)\right| \\
& \leqq\left|\frac{e^{-m y}}{\sinh y}-\frac{e^{-m x}}{\sinh x}\right|+\left|\frac{e^{-m x}}{\sinh x}\left(\frac{x^{2}}{3 !}+\frac{x^{4}}{5 !}+\frac{x^{6}}{7 !}+\cdots\right)\right|
\end{aligned}
$$

Therefore,

$$
|D| \leqq 2\left(d_{1}+d_{2}\right), \text { where } d_{1}=\int_{0}^{e}\left|\frac{e^{-m y}}{\sinh y}-\frac{e^{-m x}}{\sinh x}\right| d x
$$

and

$$
d_{2}=\int_{0}^{e} \frac{e^{-m x}}{\sinh x}\left(\frac{x^{2}}{3 !}+\frac{x^{4}}{5 !}+\frac{x^{6}}{7 !}+\cdots\right) d x
$$

We shall first consider $d_{1}$. Let $f(x)=e^{-m x} / \sinh x$, so that $d_{1}=\int_{0}^{\epsilon}|f(x)-f(y)| d x$. Since $m \geqq 1$ is assumed in all of these estimates of $g(m, n)$, we need only to consider $x$ in the range $0 \leqq x \leqq 1$ (as in Lemma 2). By the Mean Value Theorem, we have

$$
d_{1}=\int_{0}^{e}(x-y)\left|f^{\prime}(\xi)\right| d x
$$

where

$$
y \leqq \xi \leqq x
$$

Now

$$
\left|f^{\prime}(\xi)\right|=\left|\frac{e^{-m \xi}}{\sinh ^{2 \xi}}(m \sinh \xi+\cosh \xi)\right|
$$

and, since

$$
y \leqq \xi \leqq x
$$

we have

Therefore

$$
\sinh y \leqq \sinh \xi \leqq \sinh x \text { and } \cosh \xi \leqq \cosh x
$$

$$
\left|f^{\prime}(\xi)\right| \leqq \frac{e^{-m y}}{\sinh ^{2} y}(m \sinh x+\cosh x)
$$


But from Eqs. (22) through (25), we have

$$
e^{-m y} \leqq e^{-m\left(x-x^{3} / 10\right)} \leqq e^{-m(x-x / 10)}=e^{-9 m x / 10}, \quad \sinh ^{2} y \geqq \frac{81 x^{2}}{100} \geqq \frac{5 x^{2}}{5} ，
$$

and

$$
m \sinh x+\cosh x \leqq \frac{6 m x}{5}+\frac{8}{5}
$$

Therefore

$$
\left|f^{\prime}(\xi)\right| \leqq \frac{e^{-9 m x / 10}(6 m x+8)}{4 x^{2}}
$$

and, hence

$$
d_{1} \leqq \int_{0}^{\epsilon} \frac{x^{3}}{10} \frac{e^{-9 m x / 10}(6 m x+8)}{4 x^{2}} d x=\frac{3 m}{20} \int_{0}^{\epsilon} x^{2} e^{-9 m x / 10} d x+\frac{1}{5} \int_{0}^{\epsilon} x e^{-9 m x / 10} d x .
$$

Substituting $\epsilon=m^{-1 / 3}$ then, after dropping negative valued terms, there results

$$
d_{1} \leqq \frac{160}{243 m^{2}},
$$

$m \geqq 1$.

In considering $d_{2}$, let

$$
\frac{x^{2}}{3 !}+\frac{x^{4}}{5 !}+\frac{x^{6}}{7 !}+\cdots=\frac{x^{2}}{3 !} S
$$

where

$$
S=1+\frac{x^{2}}{4 \cdot 5}+\frac{x^{4}}{4 \cdot 5 \cdot 6 \cdot 7}+\cdots \leqq 1+\frac{x^{2}}{4^{2}}+\frac{x^{4}}{4^{4}}+\cdots=\frac{16}{16-x^{2}} \leqq \frac{16}{15} .
$$

Hence,

$$
\frac{x^{2} S}{3 !} \leqq \frac{8 x^{2}}{45} \leqq \frac{x^{2}}{5}
$$

Furthermore, by Eqs. (22) and (23), $\sinh x \geqq 9 x / 10$ and, therefore, $d_{2} \leqq 2 / 9 \int_{0}^{\epsilon} x e^{-m x} d x \leqq 2 / 9 m^{2}, m \geqq 1$ (after negative terms have been dropped and $\epsilon=m^{-1 / 3}$ has been substituted). Combining the above results, we obtain

$$
|D| \leqq 2\left(d_{1}+d_{2}\right) \leqq \frac{2}{m^{2}}\left(\frac{160}{243}+\frac{2}{9}\right) \leqq \frac{9}{5 m^{2}}, \quad m \geqq 1 .
$$

5. Proof of Theorem 1. Letting

$$
F(m)=2 m^{-2 / 3} \exp \left[-m^{2 / 3}\right]+\frac{20 \pi}{9} m^{1 / 3} \exp \left[-\frac{9 m^{2 / 3}}{10}\right]+\frac{9}{5 m^{2}}
$$

and

$$
G(m)=m^{2} F(m)
$$

one notes that

$$
\frac{d G}{d m}<0 \text { for } \quad m \geqq 8
$$


Therefore, $G(m)$ is a monotonic decreasing function for $m \geqq 8$ i.e.,

$$
G(8)>G(m), \quad \quad m=9,10,11, \text { etc. }
$$

One may also verify that

$$
\max [G(1), G(2), \cdots, G(8)]=G(8)=26 \cdot 80 .
$$

Hence

$$
|B|+|C|+|D| \leqq \frac{G(8)}{m^{2}}=\frac{26 \cdot 80}{m^{2}} .
$$

Combining this with Eqs. (17) and (19), one obtains

$$
-\frac{G(8)}{m^{2}} \leqq 2 \pi g(m, n)-\log k-\frac{3}{2} \log 2-\gamma \leqq \frac{1}{24 m^{2}}+\frac{G(8)}{m^{2}}
$$

where

$$
m \geqq n \quad \text { and } \quad m=1,2,3, \cdots .
$$

Finally, since $m^{2}+n^{2} \geqq 2 n^{2}$, we are led to

$$
-\frac{53 \cdot 60}{k^{2}} \leqq 2 \pi g(m, n)-\log k-\frac{3}{2} \log 2-\gamma \leqq \frac{53 \cdot 60}{k^{2}}+\frac{1}{12 k^{2}} .
$$

This completes the verification of estimate (11a). As for estimate (11b), it follows from the definition

$$
D g_{P}(Q)=\frac{\delta_{P, Q}}{h^{2}}=\frac{1}{h^{2}} \quad \text { if } P=Q ; \quad 0 \text { if } P \neq Q
$$

and from the fact that

$$
g_{P}(Q)=g(m, n)+\frac{1}{2 \pi} \log h .
$$

To verify the remark following estimates (11), we shall make use of Eq. (18) by taking more terms in that series. The estimates for $|B|,|C|$, and $|D|$ remain unchanged, while only $A^{*}$ changes. For example, by taking an additional term in Eq. (18), one is led to

$$
\frac{1}{24 m^{2}}-\frac{7}{960 m^{4}} \leqq A^{*}-\log k-\frac{3}{2} \log 2-\gamma \leqq \frac{1}{24 m^{2}}
$$

and, hence

$$
\begin{aligned}
\frac{1}{24 k^{2}}-\frac{53 \cdot 60}{k^{2}}-\frac{7}{190 k^{4}} & \leqq 2 \pi g(a, b)-\log k-\frac{3}{2} \log 2-\gamma \\
& \leqq \frac{53 \cdot 60}{k^{2}}+\frac{1}{12 k^{2}} .
\end{aligned}
$$

This process of taking more terms in relation (18) may be continued indefinitely.

Space and Information Systems Division

North American Aviation, Inc.

Downey, California 
1. R. J. Dufrin, "Discrete potential theory," Duke Math. J., v. 20, 1953, pp. 233-251. MR 16, 1119.

2. R. J. Dufrin \& E. P. Shelly, "Difference equations of polyharmonic type," Duke Math. J., v. 25, 1958, pp. 209-238. MR $20 * 5374$.

3. R. J. DUFFin \& D. H. ShafFer, "Asymptotic expansion of double Fourier transforms," Duke Math. J., v. 27, 1960, pp. 581-596. MR 22 \%8280.

4. B. Friedman, Principles and Techniques of Applied Mathematics, Wiley, New York, 1960.

5. L. B. W. Jolley, Summation of Series, 2nd rev. ed., Dover, New York, 1961. MR 24 * B511.

6. W. H. McCrea \& F. J. W. Whipple, "Random paths in two and three dimensions," Proc. Roy. Soc. Edinburgh, v. 60, 1939. 